\title{
Error Estimates for the Numerical Identification of a Variable Coefficient*
}

\author{
By Richard S. Falk
}

\begin{abstract}
Error estimates are derived for the approximate identification of an unknown transmissivity coefficient in a partial differential equation describing a model problem in groundwater flow. The approximation scheme considered determines the coefficient by least squares fitting of the observed pressure data.
\end{abstract}

1. Introduction. In this paper we wish to present an error analysis of a common numerical scheme used in the identification of parameters in distributed systems. Specifically, we shall concern ourselves here with a model problem in groundwater flow. The problem is to identify a spatially varying transmissivity coefficient $a(x)$ from observations of the piezometric head $u(x)$ in a two-dimensional static aquifer $\Omega$, where $a$ and $u$ are related by the equations

$$
\begin{gathered}
-\operatorname{div}(a \nabla u)=f \quad \text { in } \Omega, \\
a \frac{\partial u}{\partial n}=g, \quad \text { on } \partial \Omega,
\end{gathered}
$$

and $f$ and $g$ are given functions satisfying the compatibility condition $\int_{\Omega} f d x+$ $\int_{\partial \Omega} g d s=0$.

If $a$ cannot be measured directly, but it is possible to obtain an approximate measurement $z$ of $u$, then a common approach to the approximate determination of $a$ (see for example [6]) is to solve the problem

$\left(\mathrm{P}_{h}\right)$ Find $a_{h} \in K_{h}$ such that

$$
J\left(a_{h}\right)=\inf _{\bar{b} \in K_{h}} J(\bar{b})
$$

where $J(\bar{b})=\left\|u_{h}(\bar{b})-z\right\|_{L_{2}(\Omega)}^{2}$,

$$
K_{h}=\left\{\bar{b} \in T_{h}: 0<c_{0} \leqslant \bar{b} \leqslant c_{1}\right\}
$$

(with $c_{0}, c_{1}$ given a priori bounds on the transmissivity), and $u_{h}(\bar{b}) \in S_{h}$ is defined by

$$
\int_{\Omega} \bar{b} \nabla u_{h}(\bar{b}) \nabla v_{h} d x=\int_{\partial \Omega} g v_{h} d s+\int_{\Omega} f v_{h} d x,
$$

for all $v_{h} \in S_{h}$, and

$$
\int_{\Omega} u_{h}(\bar{b}) d x=\int_{\Omega} z d x
$$

Received November 26, 1979.

1980 Mathematics Subject Classification. Primary 35R30, 65M30, 65N15, 65N30.

Key words and phrases. Inverse problem, identification problem.

*This work was supported by NSF grant MCS78-02737. 
In the above $T_{h}$ and $S_{h}$ are finite-dimensional subspaces of $L_{2}(\Omega)$ and $H^{1}(\Omega)$, respectively.

Our main concern in this paper will be to derive error estimates for such a procedure under conditions that guarantee the inverse problem of identifying $a(x)$ is well-posed. To see that in general the inverse problem is not well-posed, observe from (1) that if $\nabla u$ vanishes on some subdomain of $\Omega$, one can have nonuniqueness of the transmissivity coefficient.

For this model problem it is also possible to construct approximation schemes which solve directly for $a$ using Eq. (1) viewed as a hyperbolic equation for $a$. An approximation scheme using Galerkin's method is proposed in Frind and Pinder [4], although no error analysis is given, and a finite difference scheme is proposed and analyzed in Richter [7]. Further references for various approaches to this problem can be found in the paper of Yoon and Yeh [9].

We also note that although the approximate problem $\left(\mathrm{P}_{h}\right)$ is based on viewing the underlying equation (1) as an elliptic equation for the pressure $u(x)$, some of the analysis will be based on viewing (1) as a hyperbolic equation for the transmissivity $a$. In this regard, the work of Lesaint [5] has been useful.

An outline of the paper is as follows. In Section 2 we define the notation to be used and state the conditions under which our main results will be obtained. Section 3 contains the derivation of the error estimates for the approximation scheme defined in Section 1. Finally in Section 4 we give a brief description of a method for solving the approximate problem.

2. Notation and Preliminaries. For $\Omega$ a bounded domain in $\mathbf{R}^{2}$ and $k$ a nonnegative integer we shall denote by $W^{k, p}(\Omega)$ the usual Sobolev spaces of functions defined on $\Omega$ with norms

$$
\|u\|_{k, p, \Omega}=\left\{\sum_{|j|=0}^{k}\left\|D^{j} u\right\|_{L_{p}(\Omega)}^{p}\right\}^{1 / p}, \quad 1 \leqslant p<\infty
$$

and

$$
\|u\|_{k, \infty, \Omega}=\sum_{|j|=0}^{k}\left\|D^{j} u\right\|_{L_{\infty}(\Omega)} .
$$

We further denote by $H^{k}(\Omega)$ the space $W^{k .2}(\Omega)$ and will use the notation $\|u\|_{k, \Omega}$ to denote the norms in $H^{k}$. In most cases the intended domain will be clear from the context and so the subscript $\Omega$ in the norm will be dropped.

We shall also use the notation $(\cdot, \cdot)$ to denote the $L_{2}$ inner product in $\Omega$, and $\langle\cdot, \cdot\rangle$ to denote $L_{2}$ inner product on $\Gamma=\partial \Omega$. For future reference we note that using (1), (2) the true piezometric head $u$ is related to the transmissivity $a$ by the variational equation

$$
(a \nabla u, \nabla v)=(f, v)+\langle g, v\rangle \quad \forall v \in H^{1}(\Omega) .
$$

We shall normalize $u$ to also satisfy

$$
\int_{\Omega}(u-z) d x=0 .
$$


To define the finite-dimensional subspaces used in the approximation scheme, we let $\Delta_{h}, 0<h<1$, be a triangulation of $\bar{\Omega}$ with triangles $T$ of diameter less than or equal to $h$. If the boundary is curved, we shall use triangles at the boundary with one edge replaced by a segment of the boundary. We assume the family $\left\{\Delta_{h}\right\}$ is regular and quasi-uniform. For $r \geqslant 1$ a fixed integer we then define

$$
S_{h}^{r}=\left\{v \in C^{0}(\bar{\Omega}):\left.v\right|_{T} \in P_{r} \forall T \in \Delta_{h}\right\},
$$

where $P_{r}$ is the space of polynomials of degree $r$ or less in the variables $x_{1}$ and $x_{2}$. For information about the properties of such spaces we refer the reader to [2] and to [8] for the case of a curved boundary.

The error estimates for the approximation scheme $\left(\mathrm{P}_{h}\right)$ will be derived under the following two assumptions about the true piezometric head $u$.

There exists a constant unit vector $\vec{\nu}$ and a constant $\sigma>0$ such that $\nabla u \cdot \vec{\nu} \geqslant \sigma>0 \forall x \in \Omega$,

and

(A2) $u \in W^{r+3, \infty}(\Omega)$ and $\Gamma_{1}=\left\{x \in \Gamma: \frac{\partial u}{\partial n}>0\right\} \in C^{r+2} \quad(r \geqslant 1)$.

We now make some remarks about and examine the implications of these assumptions. (Al) is a physical hypothesis stating that there is always some flow in the $\vec{\nu}$ direction. Along with a regularity assumption on $u$, it is sufficient to guarantee uniqueness of the inverse problem for the determination of $a(x)$.

LEMma 1. Assume condition (A1) holds and that $u \in W^{2, \infty}(\Omega)$. Then there is at most one coefficient $a(x) \in H^{1}(\Omega)$ and satisfying (6).

Proof. Assume $b$ is another such coefficient. Then subtracting equations we get

$$
((a-b) \nabla u, \nabla v)=0 \text { for all } v \in H^{1}(\Omega),
$$

which further impies that $(a-b) \partial u / \partial n=0$ on $\Gamma$. Choosing $v=e^{-2 k \vec{x} \cdot \vec{v}}(b-a)$, where $k>\|\Delta u\|_{0, \infty} /(2 \sigma)$, and integrating by parts, one can show (see the proof of Theorem 1 with $\rho=1$ ) that

$$
\begin{aligned}
((a-b) \nabla u, \nabla v)= & -\frac{1}{2}\left\langle e^{-2 k \vec{x} \cdot \vec{v}} \frac{\partial u}{\partial n},[a-b]^{2}\right\rangle \\
& +\left([a-b]^{2} e^{-2 k \vec{x} \cdot \vec{v}}, k \nabla u \cdot \vec{v}+\frac{1}{2} \Delta u\right) .
\end{aligned}
$$

Applying condition (Al) and the fact that $(a-b) \partial u / \partial n=0$ on $\Gamma$, it easily follows that $((a-b) \nabla u, \nabla v) \geqslant \bar{\tau}\|a-b\|_{0}^{2}$ for some $\bar{\tau}>0$. Hence $\|a-b\|_{0}=0$ and so $a=b$.

Hypothesis (A2) is a technical one giving sufficient conditions for the validity of the following result, which we use later in the derivation of the error estimates.

Lemma 2. Assume hypotheses (A1) and (A2) hold. Then given $\tau>0$, there exists a function $\rho \in W^{r+2, \infty}(\Omega)$ satisfying $\rho=0$ on $\Gamma_{1}$, and $\rho\left[k \nabla u \cdot \vec{v}+\frac{1}{2} \Delta u\right]-\frac{1}{2} \nabla \rho \cdot \nabla u$ $\geqslant \tau>0$, where $k=\|\Delta u\|_{0, \infty} /(2 \sigma)$.

Proof. Let $\rho$ be the solution of the Cauchy problem

$$
\begin{aligned}
\nabla \rho \cdot \nabla u & =-2 \tau & & \text { in } \Omega, \\
\rho & =0 & & \text { on } \Gamma_{1} .
\end{aligned}
$$


Since $\partial u / \partial n>0$ on $\Gamma_{1}, \Gamma_{1}$ is not characteristic and, by (A1), $|\nabla u| \neq 0$. Hence, for $\Gamma_{1}$ and $u$ sufficiently smooth, we get a unique smooth solution of this initial value problem. In fact by (A1) we can take as local coordinates $u$ and $v$, where $v$ is a coordinate along the lines $u=$ constant. Writing $\Gamma_{1}$ in the form $u=G(v)$, an easy computation shows

$$
\rho(x, y) \equiv P(u, v)=\int_{G(v)}^{u} \frac{-2 \tau}{\left(u_{x}^{2}+u_{y}^{2}\right)(s, v)} d s .
$$

Using this formula we see that $\rho \geqslant 0$ in $\Omega$. Differentiating the formula then shows that condition (A2) is sufficient to guarantee the desired smoothness of $\rho$.

3. Error Estimates. In order to derive our main result we will need an estimate of how well one can expect to approximate the true piezometric head $u$ by functions of the form $u_{h}(\bar{b})$ (defined by (4), (5)), for $\bar{b} \in K_{h}$. That estimate is derived in the following:

LEMmA 3. Suppose that $a \in H^{r+1}(\Omega)$ and $u \in H^{r+2}(\Omega)(r \geqslant 1)$ satisfy (6)-(7) and that

$$
0<c_{0}<\min _{x \in \bar{\Omega}} a(x) \leqslant \max _{x \in \bar{\Omega}} a(x)<c_{1} .
$$

Then if $K_{h}$ is defined by (3) and $u_{h}(\bar{b})$ is defined by (4), (5) with $T_{h}=S_{h}^{r}$ and $S_{h}=S_{h}^{r+1}$, we have for all $h$ sufficiently small that

$$
\inf _{\bar{b} \in K_{h}}\left\|u_{h}(\bar{b})-u\right\|_{0} \leqslant C h^{r+2},
$$

where $C$ depends on $c_{0}, c_{1},\|a\|_{r+1}$, and $\|u\|_{r+2}$, but is independent of $h$.

Proof. Let $b$ denote the $L_{2}$ projection of $a$ into $S_{h}^{r}$. Then, by a result of [3], and standard approximation results, we have, for all $1 \leqslant p \leqslant \infty$,

$$
\|a-b\|_{0, p} \leqslant C \inf _{\bar{b} \in S_{h}^{r}}\|a-\bar{b}\|_{0, p} \leqslant C h^{r+1}\|a\|_{r+1, p} .
$$

Hence, for all $x \in \bar{\Omega}$,

$$
a-C h^{r+1}\|a\|_{r+1, \infty} \leqslant b \leqslant a+C h^{r+1}\|a\|_{r+1, \infty}
$$

and so

$$
\min _{x \in \bar{\Omega}} a(x)-C h^{r+1}\|a\|_{r+1, \infty} \leqslant b \leqslant \max _{x \in \bar{\Omega}} a(x)+C h^{r+1}\|a\|_{r+1, \infty} .
$$

Thus by the definition of $c_{0}$ and $c_{1}, b \in K_{h}$ for $h$ sufficiently small.

Now, since $b \in K_{h}$, we have

$$
\inf _{\bar{b} \in K_{h}}\left\|u_{h}(\bar{b})-u\right\|_{0} \leqslant\left\|u_{h}(b)-u\right\|_{0} .
$$

Using Eqs. (4) and (6), we have for all $v_{h} \in S_{h}^{r+1}$ that

$$
\begin{aligned}
(b \nabla & {\left.\left[u_{h}(b)-u\right], \nabla v_{h}\right) } \\
& =\left(b \nabla u_{h}(b), \nabla v_{h}\right)-\left([b-a] \nabla u, \nabla v_{h}\right)-\left(a \nabla u, \nabla v_{h}\right) \\
& =-\left([b-a] \nabla u, \nabla v_{h}\right) .
\end{aligned}
$$

Letting $u_{I}$ denote the interpolate of $u$ in $S_{h}^{r+1}$, we also get for all $v_{h} \in S_{h}^{r+1}$ that

$$
\left(b \nabla\left[u_{h}(b)-u_{I}\right], \nabla v_{h}\right)=-\left([b-a] \nabla u, \nabla v_{h}\right)-\left(b \nabla\left[u_{I}-u\right], \nabla v_{h}\right) \text {. }
$$


Choosing $v_{h}=u_{h}(b)-u_{I}$, we obtain

$$
\left\|\nabla\left[u_{h}(b)-u_{I}\right]\right\|_{0} \leqslant\left[c_{1}\left\|\nabla\left(u_{I}-u\right)\right\|_{0}+\|b-a\|_{0}\|\nabla u\|_{0, \infty}\right] / c_{0} .
$$

Hence applying standard approximation results,

$$
\begin{aligned}
\left\|\nabla\left[u_{h}(b)-u\right]\right\|_{0} & \leqslant C\left[\left\|\nabla\left(u_{I}-u\right)\right\|_{0}+\|b-a\|_{0}\right] \\
& \leqslant C h^{r+1}\left[\|u\|_{r+2}+\|a\|_{r+1}\right] .
\end{aligned}
$$

To estimate $\left\|u_{h}(b)-u\right\|_{0}$ we define $\phi$ to be the solution of

$$
\begin{array}{rlrl}
-\operatorname{div}(a \nabla \phi) & =u_{h}(b)-u & & \text { in } \Omega, \\
\frac{\partial \phi}{\partial n} & =0 & & \text { on } \Gamma, \\
\int_{\Omega} \phi d x & =0, &
\end{array}
$$

and use duality. Then for all $\phi_{h} \in S_{h}^{r+1}$

$$
\begin{aligned}
\| u_{h}(b) & -u \|_{0}^{2}=\left(a \nabla\left[u_{h}(b)-u\right], \nabla \phi\right) \\
= & \left([a-b] \nabla\left[u_{h}(b)-u\right], \nabla \phi\right)+\left(b \nabla\left[u_{h}(b)-u\right], \nabla\left[\phi-\phi_{h}\right]\right) \\
& +\left(b \nabla\left[u_{h}(b)-u\right], \nabla \phi_{h}\right) .
\end{aligned}
$$

Now, using (11) and the fact that $(b-a, \gamma)=0$ for all $\gamma \in S_{h}^{r}$, we get for all $\phi_{h} \in S_{h}^{r+1}$ and $\gamma \in S_{h}^{r}$ that

$$
\begin{aligned}
\left(b \nabla\left[u_{h}(b)-u\right], \nabla \phi_{h}\right) & =-\left([b-a] \nabla u, \nabla \phi_{h}\right) \\
& =-\left([b-a] \nabla u, \nabla\left[\phi_{h}-\phi\right]\right)-([b-a], \nabla u \cdot \nabla \phi-\gamma) .
\end{aligned}
$$

Hence, for all $\phi_{h} \in S_{h}^{r+1}$ and $\gamma \in S_{h}^{r}$, we get

$$
\begin{aligned}
\left\|u_{h}(b)-u\right\|_{0}^{2} \leqslant & \|a-b\|_{0, \infty}\left\|\nabla\left[u_{h}(b)-u\right]\right\|_{0}\|\nabla \phi\|_{0} \\
& +\|b\|_{0, \infty}\left\|\nabla\left[u_{h}(b)-u\right]\right\|_{0}\left\|\nabla\left(\phi-\phi_{h}\right)\right\|_{0} \\
& +\|b-a\|_{0}\|\nabla u\|_{0, \infty}\left\|\nabla\left(\phi_{h}-\phi\right)\right\|_{0} \\
& +\|b-a\|_{0}\|\nabla u \cdot \nabla \phi-\gamma\|_{0} .
\end{aligned}
$$

Since $\|\phi\|_{2} \leqslant C\left\|u_{h}(b)-u\right\|_{0}$, we get using (9), (12), and standard approximation results that

$$
\left\|u_{h}(b)-u\right\|_{0} \leqslant C h^{r+2}
$$

where $C$ depends on $c_{0}, c_{1},\|u\|_{r+2}$ and $\|a\|_{r+1}$, but is independent of $h$. The lemma follows by combining (10) and (14).

We now derive the main result of this paper.

THEOREM 1. Suppose that assumptions (A1) and (A2) are satisfied, the hypotheses of Lemma 3 hold, and that

$$
\|z-u\|_{0} \leqslant \varepsilon
$$

Then, for all $h$ sufficiently small, we have

$$
\left\|a-a_{h}\right\|_{0} \leqslant C\left[h^{r}+h^{-2} \varepsilon\right],
$$

where $a_{h}$ is any solution of problem $\left(\mathrm{P}_{h}\right)$ and $C$ is a constant independent of $h$ and $\varepsilon$. 
Proof. Letting $b$ denote the $L_{2}$ projection of $a$ into $S_{h}$ and $w_{h}=u_{h}\left(a_{h}\right)$, we easily obtain from Eqs. (4) and (6) that for all $v_{h} \in S_{h}^{r+1}$

$$
\begin{aligned}
\left(\left[b-a_{h}\right] \nabla u, \nabla v_{h}\right)= & \left([b-a] \nabla u, \nabla v_{h}\right)+\left(a \nabla u, \nabla v_{h}\right) \\
& -\left(a_{h} \nabla\left[u-w_{h}\right], \nabla v_{h}\right)-\left(a_{h} \nabla w_{h}, \nabla v_{h}\right) \\
= & \left([b-a] \nabla u, \nabla v_{h}\right)-\left(a_{h} \nabla\left[u-w_{h}\right], \nabla v_{h}\right) .
\end{aligned}
$$

Hence, for all $v \in H^{1}(\Omega)$ and all $v_{h} \in S_{h}^{r+1}$,

(16) $-\left(\left[b-a_{h}\right] \nabla u, \nabla v\right)=\left(\left[b-a_{h}\right] \nabla u, \nabla\left[v_{h}-v\right]\right)$

$$
-\left([b-a] \nabla u, \nabla v_{h}\right)+\left(a_{h} \nabla\left[u-w_{h}\right], \nabla v_{h}\right) \text {. }
$$

Now let $\vec{\nu}$ be a constant unit vector satisfying (Al) and $\rho$ a function satisfying the conclusions of Lemma 2.

Choosing $v=\rho e^{-2 k \vec{x} \cdot \vec{v}}\left[b-a_{h}\right]$ in (16), we have

$$
\nabla v=e^{-2 k \vec{x} \cdot \vec{v}}\left[b-a_{h}\right] \nabla \rho+e^{-2 k \vec{x} \cdot \vec{v}} \rho\left\{\nabla\left(b-a_{h}\right)-2 k \vec{\nu}\left(b-a_{h}\right)\right\},
$$

and so

$$
\begin{aligned}
-\left(\left[b-a_{h}\right] \nabla u, \nabla v\right)= & -\left(e^{-2 k \vec{x} \cdot \vec{v}}\left[b-a_{h}\right]^{2}, \nabla u \cdot \nabla \rho\right) \\
& +\left(e^{-2 k \vec{x} \cdot \vec{v}}\left[b-a_{h}\right]^{2}, 2 k \rho \nabla u \cdot \vec{v}\right) \\
& -\frac{1}{2}\left(e^{-2 k \vec{x} \cdot \vec{v}} \rho \nabla u, \nabla\left[b-a_{h}\right]^{2}\right) .
\end{aligned}
$$

Now

$$
\begin{aligned}
-\frac{1}{2}\left(e^{-2 k \vec{x} \cdot \vec{v}} \rho \nabla\right. & \left.u, \nabla\left[b-a_{h}\right]^{2}\right) \\
= & -\frac{1}{2}\left\langle e^{-2 k \vec{x} \cdot \vec{v}} \rho \frac{\partial u}{\partial n},\left[b-a_{h}\right]^{2}\right\rangle \\
& +\frac{1}{2}\left(\left[b-a_{h}\right]^{2}, e^{-2 k \vec{x} \cdot \vec{v}}\{\rho \Delta u+\nabla u \cdot \nabla \rho-2 k \rho \vec{\nu} \cdot \nabla u\}\right),
\end{aligned}
$$

and thus

$$
\begin{aligned}
-\left(\left[b-a_{h}\right] \nabla u, \nabla v\right)= & -\frac{1}{2}\left\langle e^{-2 k \vec{x} \cdot \vec{v}} \rho \frac{\partial u}{\partial n},\left[b-a_{h}\right]^{2}\right\rangle \\
& -\frac{1}{2}\left(e^{-2 k \vec{x} \cdot \vec{v}}\left[b-a_{h}\right]^{2}, \nabla u \cdot \nabla \rho\right) \\
& +\left(\left[b-a_{h}\right]^{2} e^{-2 k \vec{x} \cdot \vec{v}} \rho, k \nabla u \cdot \vec{v}+\frac{1}{2} \Delta u\right) .
\end{aligned}
$$

Applying Lemma 2, it easily follows that

$$
-\left(\left[b-a_{h}\right] \nabla u, \nabla v\right) \geqslant \bar{\tau}\left\|b-a_{h}\right\|_{0}^{2}, \quad \text { where } \bar{\tau}=\tau \min _{x \in \bar{\Omega}} e^{-2 k \vec{x} \cdot \vec{\nu}} .
$$

To bound the right side of (16) we set $v_{h}$ equal to the interpolate of $v$ in $S_{h}^{r+1}$ and estimate $\left\|\nabla\left(v_{h}-v\right)\right\|_{0}$.

Letting $T$ denote an arbitrary triangle of the triangulation $\Delta_{h}$ of $\Omega$, we have by standard approximation theory that

$$
\left\|\nabla\left(v-v_{h}\right)\right\|_{0}^{2}=\sum_{T \in \Delta_{h}}\left\|\nabla\left(v-v_{h}\right)\right\|_{0, T}^{2} \leqslant \sum_{T \in \Delta_{h}}\left[C h^{r+1}\|v\|_{r+2, T}\right]^{2} .
$$


Now, since $v=\rho e^{-2 k \vec{x} \cdot \vec{v}}\left[b-a_{h}\right], \rho \in W^{r+2, \infty}$, and $\left.\left[b-a_{h}\right]\right|_{T} \in P_{r}(T)$,

$$
\|v\|_{r+2, T} \leqslant C(\rho)\left\|b-a_{h}\right\|_{r, T} .
$$

Combining (18), (19), and the inverse property

$$
\left\|b-a_{h}\right\|_{r, T} \leqslant C h^{-r}\left\|b-a_{h}\right\|_{0, T},
$$

we obtain

$$
\left\|\nabla\left(v-v_{h}\right)\right\|_{0} \leqslant C h\left\|b-a_{h}\right\|_{0} .
$$

Again using the inverse property, we get

(21) $\|\nabla v\|_{0} \leqslant\left\|e^{-2 k \vec{x} \cdot \vec{v}} \nabla \rho\right\|_{0, \infty}\left\|b-a_{h}\right\|_{0}$

$$
\begin{aligned}
& +\left\|e^{-2 k \vec{x} \cdot \vec{\nu}} 2 \rho k \vec{\nu}\right\|_{0, \infty}\left\|b-a_{h}\right\|_{0}+\left\|e^{-2 k \vec{x} \cdot \vec{v}} \rho\right\|_{0, \infty}\left\|\nabla\left(b-a_{h}\right)\right\|_{0} \\
\leqslant & C h^{-1}\left\|b-a_{h}\right\|_{0} .
\end{aligned}
$$

Combining (20) and (21), we also have

$$
\left\|\nabla v_{h}\right\|_{0} \leqslant\left\|\nabla\left(v_{h}-v\right)\right\|_{0}+\|\nabla v\|_{0} \leqslant C h^{-1}\left\|b-a_{h}\right\|_{0} .
$$

Using (20), we get

$$
\begin{aligned}
\left(\left[b-a_{h}\right] \nabla u, \nabla\left[v_{h}-v\right]\right) & \leqslant\left\|b-a_{h}\right\|_{0}\|\nabla u\|_{0, \infty}\left\|\nabla\left(v_{h}-v\right)\right\|_{0} \\
& \leqslant C h\left\|b-a_{h}\right\|_{0}^{2} .
\end{aligned}
$$

From (22) and standard approximation properties we have

$$
\begin{aligned}
-\left([b-a] \nabla u, \nabla v_{h}\right) & \leqslant\|b-a\|_{0}\|\nabla u\|_{0, \infty}\left\|\nabla v_{h}\right\|_{0} \\
& \leqslant C h^{-1}\left\|b-a_{h}\right\|_{0} h^{r+1}\|a\|_{r+1} \leqslant C h^{r}\left\|b-a_{h}\right\|_{0} .
\end{aligned}
$$

Applying (22) and the fact that $\left\|a_{h}\right\|_{0, \infty} \leqslant c_{1}$, we also obtain

$$
\begin{aligned}
\left(a_{h} \nabla\left[u-w_{h}\right], \nabla v_{h}\right) & \leqslant\left\|a_{h}\right\|_{0, \infty}\left\|\nabla\left(u-w_{h}\right)\right\|_{0}\left\|\nabla v_{h}\right\|_{0} \\
& \leqslant C h^{-1}\left\|b-a_{h}\right\|_{0}\left\|\nabla\left(u-w_{h}\right)\right\|_{0} .
\end{aligned}
$$

Letting $u_{I}$ denote the interpolate of $u$ in $S_{h}^{r+1}$, we get using the inverse properties of $S_{h}^{r+1}$ that

$$
\begin{aligned}
\left\|\nabla\left(u-w_{h}\right)\right\|_{0} \leqslant & \left\|\nabla\left(u-u_{I}\right)\right\|_{0}+\left\|\nabla\left(u_{I}-w_{h}\right)\right\|_{0} \\
\leqslant & \left\|\nabla\left(u-u_{I}\right)\right\|_{0}+C h^{-1}\left\|u_{I}-w_{h}\right\|_{0} \\
\leqslant & \left\|\nabla\left(u-u_{I}\right)\right\|_{0}+C h^{-1}\left\|u_{I}-u\right\|_{0} \\
& +C h^{-1}\|u-z\|+C h^{-1}\left\|z-w_{h}\right\|_{0} .
\end{aligned}
$$

Recalling that $w_{h}=u_{h}\left(a_{h}\right)$, we then have by the definition of $a_{h}$, Lemma 3 , and hypothesis (15) that

$$
\begin{aligned}
\left\|z-w_{h}\right\|_{0} & =\left\|z-u_{h}\left(a_{h}\right)\right\|_{0}=\inf _{\bar{b} \in K_{h}}\left\|z-u_{h}(\bar{b})\right\|_{0} \\
& \leqslant \inf _{\bar{b} \in K_{h}}\left\|u-u_{h}(\bar{b})\right\|_{0}+\|u-z\|_{0} \leqslant \varepsilon+C h^{r+2} .
\end{aligned}
$$

Combining (25), (26), and (27), and applying standard approximation results, we get

$$
\begin{aligned}
& \left(a_{h} \nabla\left[u-w_{h}\right], \nabla v_{h}\right) \\
& \quad \leqslant C h^{-1}\left\|b-a_{h}\right\|_{0}\left\{C h^{r+1}+C h^{-1} \varepsilon\right\} \leqslant C\left[h^{r}+h^{-2} \varepsilon\right]\left\|b-a_{h}\right\|_{0} .
\end{aligned}
$$


Combining (23), (24), and (28), the right side of (16) is bounded by

$$
C h\left\|b-a_{h}\right\|_{0}^{2}+C\left[h^{r}+h^{-2} \varepsilon\right]\left\|b-a_{h}\right\|_{0} .
$$

It now easily follows from (16), (17), and (29) that, for $h$ sufficiently small,

$$
\left\|b-a_{h}\right\|_{0} \leqslant C\left[h^{r}+h^{-2} \varepsilon\right]
$$

and so finally

$$
\left\|a-a_{h}\right\|_{0} \leqslant\|a-b\|_{0}+\left\|b-a_{h}\right\|_{0} \leqslant C\left[h^{r}+h^{-2} \varepsilon\right] .
$$

COROLlaRY. If $z=u_{I}$, the interpolate of $u$ in $S_{h}^{r+1}$, then for $h$ sufficiently small $\left\|a-a_{h}\right\|_{0} \leqslant C h^{r}$ for some constant $C$ independent of $h$.

The reader should note that the error estimate obtained in Theorem 1 (with $\varepsilon=0$ ) is one power of $h$ less than is possible by the best approximation of $a$ by elements of the subspace $S_{h}^{r}$. Although we believe the result to be optimal for the two-dimensional problem considered, we now show how the result can be improved for the one-dimensional analogue of this problem.

We thus consider the problem where $\Omega=[0,1]$. Besides obtaining a better error estimate, the result in one dimension will be more general since we can now allow a wider choice of subspaces $T_{h}$ and $S_{h}$.

For $0<h<1$ we now let $\Delta_{h}$ be a quasi-uniform partition of $[0,1]$ into subintervals $I$ of length less than or equal to $h$. For $r \geqslant 0$ and $k \geqslant-1$ integers we then define

$$
T_{h}^{r, k}=\left\{b \in C^{k}(\bar{\Omega}):\left.b\right|_{I} \in P_{r} \forall I \in \Delta_{h}\right\},
$$

where $P_{r}$ is the space of polynomials of degree $r$ or less and $C^{-1}(\bar{\Omega})$ denotes no interelement continuity requirement.

We now derive the following improved estimate.

TheOREM 2. Suppose that $a \in H^{r+1}(\Omega), u \in H^{r+2}(\Omega)(r \geqslant 0)$ satisfy (6)-(7),

$$
D u \geqslant \gamma>0 \text { for some constant } \gamma
$$

(the analogue of (A1) where $D=d / d x$ ), and that hypothesis (8) of Lemma 3 is satisfied. Then if $a_{h}$ is a solution of problem $\left(\mathrm{P}_{h}\right)$ with $T_{h}=T_{h}^{r, k}$ and $S_{h}=T_{h}^{s, l}$ where $r \geqslant 0, k \geqslant-1, s \geqslant r+1$ and $0 \leqslant l \leqslant k+1$, and $\|u-z\|_{0} \leqslant \varepsilon$, we have for $h$ sufficiently small that

$$
\left\|a-a_{h}\right\|_{0} \leqslant C\left[h^{r+1}+h^{-1} \varepsilon\right],
$$

where $C$ is a constant independent of $h$ and $\varepsilon$.

Proof. Letting $b$ denote the $L_{2}$ projection of $a$ into $T_{h}^{r, k}$ and $w_{h}=u_{h}\left(a_{h}\right)$, we easily obtain from Eqs. (4) and (6) that for all $v_{h} \in T_{h}^{s, l}$

$$
\left(\left[b-a_{h}\right] D u, D v_{h}\right)=\left([b-a] D u, D v_{h}\right)-\left(a_{h} D\left[u-w_{h}\right], D v_{h}\right) \text {. }
$$

Observing that the choice $v_{h}=\int_{1}^{x}\left(b-a_{h}\right)(s) d s \in T_{h}^{s, l}$, we get

$$
\left(D u,\left[b-a_{h}\right]^{2}\right)=\left([b-a] D u, b-a_{h}\right)-\left(a_{h} D\left[u-w_{h}\right], b-a_{h}\right),
$$

and so applying (30) it follows that

$$
\left\|b-a_{h}\right\|_{0} \leqslant\left\{\|b-a\|_{0}\|D u\|_{0, \infty}+\left\|a_{h}\right\|_{0, \infty}\left\|D\left[u-w_{h}\right]\right\|_{0}\right\} / \gamma .
$$

Since $a_{h} \in K_{h}$, we get for some constant $C$ independent of $h$ that

$$
\left\|a-a_{h}\right\|_{0} \leqslant\|a-b\|_{0}+\left\|b-a_{h}\right\|_{0} \leqslant C\left[\|a-b\|_{0}+\left\|D\left[u-w_{h}\right]\right\|_{0} .\right.
$$


Now letting $u_{I}$ denote the interpolate of $u$ in $T_{h}^{s, l}$, we get using the inverse properties of $T_{h}^{s, l}$ that

$$
\begin{aligned}
& \left\|D\left[u-w_{h}\right]\right\|_{0} \leqslant\left\|D\left[u-u_{I}\right]\right\|_{0}+\left\|D\left[u_{I}-w_{h}\right]\right\|_{0} \\
& \quad \leqslant\left\|D\left[u-u_{I}\right]\right\|_{0}+C h^{-1}\left\|u_{I}-w_{h}\right\|_{0} \\
& \quad \leqslant\left\|D\left[u-u_{I}\right]\right\|_{0}+C h^{-1}\left\|u_{I}-u\right\|_{0}+C h^{-1}\|u-z\|_{0}+C h^{-1}\left\|z-w_{h}\right\|_{0} .
\end{aligned}
$$

We next observe that the one-dimensional analogue of Lemma 3 is valid for $r \geqslant 0$ and $T_{h}$ and $S_{h}$ chosen as in the hypotheses of Theorem 2 . Hence, recalling that $w_{h}=u_{h}\left(a_{h}\right)$, we have by the definition of $a_{h}$ and Lemma 3 that

$$
\begin{aligned}
\left\|z-w_{h}\right\|_{0} & =\left\|z-u_{h}\left(a_{h}\right)\right\|_{0}=\inf _{\bar{b} \in K_{h}}\left\|z-u_{h}(\bar{b})\right\|_{0} \\
& \leqslant \inf _{\bar{b} \in K_{h}}\left\|u-u_{h}(\bar{b})\right\|_{0}+\|u-z\|_{0} \leqslant C h^{r+2}+\varepsilon .
\end{aligned}
$$

Combining (31), (32), and (33), and applying standard approximation properties, we get

$$
\left\|a-a_{h}\right\|_{0} \leqslant C\left[h^{r+1}+h^{-1} \varepsilon\right] .
$$

4. Solution of the Approximate Problem. In order to determine the approximate transmissivity coefficient $a_{h}$, we must solve problem $\left(\mathrm{P}_{h}\right)$. Writing $a_{h}=\sum_{i=1}^{m} \alpha_{i} \Phi_{i}$, where $\left\{\Phi_{i}\right\}_{i=1}^{m}$ are a basis for $S_{h}^{r}$, problem $\left(\mathrm{P}_{h}\right)$ reduces to a nonlinear programming problem to obtain the coefficients $\left\{\alpha_{i}\right\}_{i=1}^{m}$. In the case $r=1, S_{h}^{r}$ consists of piecewise linear functions, so that the constraint set $K_{h}$ reduces to the set of linear inequality constraints $c_{0} \leqslant \alpha_{i} \leqslant c_{1}, i=1, \ldots, m$.

One possibility for the resolution of this nonlinear programming problem is to use some type of gradient projection method. The steepest descent algorithm, for example, has been successfully used in work of Chavent [1], where the gradient of $J$ is computed by introducing an adjoint variable.

For each $\bar{b} \in K_{h}$ we define $p_{h}(\bar{b}) \in S_{h}^{r+1}$ as the solution of

$$
\begin{aligned}
& \left(\bar{b} \nabla p_{h}(\bar{b}), \nabla v_{h}\right)=2\left(u_{h}(\bar{b})-z, v_{h}\right) \\
& \qquad \text { for all } v_{h} \in S_{h}^{r+1} \text { and } \int_{\Omega} p_{n}(\bar{b}) d x=0 .
\end{aligned}
$$

It is then easy to check that if $\bar{b}=\sum_{i=1}^{m} \beta_{i} \Phi_{i}$ and $b=\sum_{i=1}^{m} \gamma_{i} \Phi_{i} \in S_{h}^{r}$, then

$$
J^{\prime}(\bar{b}) \cdot b=\sum_{i=1}^{m} \frac{\partial J}{\partial \beta_{i}} \gamma_{i}=-\left(b \nabla u_{h}(\bar{b}), \nabla p_{h}(\bar{b})\right) \text {. }
$$

Hence the evaluation of $J^{\prime}(\bar{b}) \cdot b$ simply requires the solution of the two linear systems of equations corresponding to Eqs. (4) and (34). References to other approaches can be found in [9].

Acknowledgements. Part of this work was performed while the author was visiting the Laboratoire Analyse Numérique at the Universite Pierre et Marie Curie and also the Département de Mathématiques et Informatique at the Université de Rennes. The author wishes to express his gratitude for their financial support. 
1. G. Chavent, "Identification of distributed parameters," in Identification and System Parameter Estimation, Proc. 3rd IFAC Sympos. (P. Eykhoff, Ed.), pp. 649-660.

2. P. Ciarlet, The Finite Element Method for Elliptic Problems, North-Holland, Amsterdam, 1978.

3. J. Douglas, JR., T. Dupont \& L. Wahlbin, "The stability in $L^{q}$ of the $L^{2}$-projection into finite element function spaces," Numer. Math., v. 23, 1975, pp. 193-197.

4. E. FRIND \& G. PINDER, "Galerkin solution of the inverse problem for aquifier transmissivity," Water Resources Research, v. 9, 1973, pp. 1397-1410.

5. P. Lesaint, "Finite element methods for symmetric hyperbolic equations," Numer. Math., v. 21, 1973. pp. 244-255.

6. M. P. Polis \& R. E. Goodson, "Parameter identification in distributed systems: A synthesizing overvicw," Proc. IEEE, v. 64, 1976, pp. 45-61.

7. G. Richter, "Numerical identification of a spatially varying diffusivity coefficient," Math. Comp., v. 36, 1981, pp. 375-386.

8. R. SCOTT, "Interpolated boundary conditions in the finite element method," SIAM J. Numer. Anal., v. 12, 1975, pp. 404-427.

9. Y. S. YOON \& W. W.-G. YEH, "Parameter identification in an inhomogeneous medium with the finite-element method," Soc. Pet. Eng. J., v. 16, 1976, pp. 217-226. 